\title{
Energy Conversion Loops for Flux-Switching PM Machine Analysis
}

\author{
E. Ilhan \\ Electromechanics and Power \\ Electronics Group (EPE) \\ Electrical Engineering \\ Eindhoven University of \\ Technology \\ Eindhoven, Netherlands \\ e.ilhan@tue.nl, \\ esinilhan@hotmail.com
}

\author{
T. E. Motoasca \\ Electromechanics and \\ Power Electronics Group \\ (EPE) \\ Electrical Engineering \\ Eindhoven University of \\ Technology \\ Eindhoven, Netherlands
}

\author{
J. J. H. Paulides \\ Electromechanics and \\ Power Electronics Group \\ (EPE) \\ Electrical Engineering \\ Eindhoven University of \\ Technology \\ Eindhoven, Netherlands
}

\author{
E. A. Lomonova \\ Electromechanics and \\ Power Electronics Group \\ (EPE) \\ Electrical Engineering \\ Eindhoven University of \\ Technology \\ Eindhoven, Netherlands
}

\begin{abstract}
Induction and synchronous machines have traditionally been the first choice of automotive manufacturers for electric/hybrid vehicles. However, these conventional machines are not able anymore to meet the increasing demands for a higher energy density due to space limitation in cars. Fluxswitching PM (FSPM) machines with their high energy density are very suitable to answer this demand. In this paper, the energy conversion loop technique is implemented on FSPM for the first time. The energy conversion technique is a powerful tool for the visualization of machine characteristics, both linear and nonlinear. Further, the technique provides insight into the torque production mechanism. A stepwise explanation is given on how to create these loops for FSPM along with the machine operation.
\end{abstract}

Keywords-energy conversion loops; flux-switching PM machines; nonlinear magnetic analysis; PM motors

\section{INTRODUCTION}

Flux-switching PM (FSPM) machines are suitable alternatives when it comes to e-mobility applications. What makes FSPM appealing for the automotive industry, among its many advantages, is its high energy density in the airgap [1], which is caused by the operation near the saturation region. This operating point results in a nonlinear behavior of the softmagnetic material used in the machine. Additionally, FSPM has a rather unconventional structure, embodying all its energy sources in the stator frame, as illustrated in Figure 1a. Due to this arrangement of sources, the double salient machine structure is a must for its operation; however, this geometry results in a non-uniform airgap. With the flux focusing effect due to the alternating magnet polarity, the double salient structure of FSPM is capable of a high torque density. It is a result of the parallel magnetic paths of the stator coils and magnets. The magnetic circuit also decreases the demagnetization probability, which is crucial for the vehicle safety.

Due to mentioned peculiarities, many researches in this area focus in modeling problems [2-7]. In these researches, analytical and numerical FSPM models are explained along with their results. This paper brings the modeling issue one step further by discussing how these models can be used to create the energy conversion loops for the FSPM. The phase flux linkage calculated by any of these methods can easily be used to create the energy conversion loops for the FSPM by following the stepwise explanation provided in this paper. Besides linear analysis also nonlinear analysis of the FSPM, which is often neglected in analytical modeling techniques $[3,5,7]$, is included in the paper. Additionally, to give more insight into machine operation, the torque calculation mechanism is explained separately. The motor quantities used in this paper, such as flux linkage, are calculated by means of the numerical Finite Element Method (FEM).

\section{ENERGY CONVERSION LOOPS FOR FSPM MACHINE}

There are multiple methods to create the energy conversion loop of an electrical machine. For constructing the energy conversion loops of the FSPM, flux linkage-current $(\lambda-i)$ characteristic is chosen. The area of the loop is proportional to the change of magnetic energy inside the machine. One energy loop corresponds to one phase flux linkage variation over one electrical cycle and the corresponding change in the instantaneous current for the same rotor position. Torque production in electrical machines mainly depends on the airgap flux density. Distinctively, FSPM does not have a rotating magnetic field in the airgap. Because of the machine saliency, there is a change of the magnetic energy in the airgap. In the FSPM, there are two separate energy sources, i.e. coils and magnets. To investigate the torque production mechanism, the sources are separately evaluated. Therefore, the energy conversion loops are constructed for the following cases: with coils and with coils\&magnets. Also the magnetic material properties are included in this work, namely linear and nonlinear. 
TABLE I. FSPM SIZE AND PARAMETERS

\begin{tabular}{|c|c|}
\hline Number of phases, $\boldsymbol{m}$ & 3 \\
\hline Rotor pole pair number, $\boldsymbol{p}$ & 10 \\
\hline Stator pole number & 12 \\
\hline Frame size & $90 \mathrm{~mm}$ \\
\hline Frame length & $25 \mathrm{~mm}$ \\
\hline Rated power & $90 \mathrm{~W}$ \\
\hline Rated torque & $2.2 \mathrm{Nm}$ \\
\hline Rated current & $10 \mathrm{~A}$ \\
\hline Rated speed & $400 \mathrm{rpm}$ \\
\hline
\end{tabular}

\section{CONSTRUCTING THE LOOPS FOR FSPM}

In the considered FSPM, with the parameters given in Table I, phase A consists of 4 coils (A1 \& A2) in 8 slots (Figure $1 \mathrm{~b}$ ). Due to the half machine periodicity, A1 and A2 coil pairs are shifted by $90^{\circ}$ mechanical. Flux linkages for one coil of phase A, for the A1-A2 pairs and for phase A are shown in Figure 2. The resulting energy conversion loops of Figure 2 are plotted in Figure 3. Because the supplied current and the magnetic flux linkage have a sinusoidal shape, the ideal loop shape is elliptic. If they have a trapezoidal form, e.g. brushless DC (BLDC) machines, the loops ideal shape is rectangular [9]. The higher flux linkage values of Figure $2 b$, compared to Figure $2 \mathrm{a}$, result in larger loops in Figure $3 \mathrm{~b}$, compared to Figure $3 \mathrm{a}$. Because the total area of these loops is proportional to the average electromagnetic torque, the loops with a larger area correspond to a higher torque $[8,10]$. The enclosed areas in Figure 3a show that the torque production in the presence of coils only is very weak compared the areas in Figure 3b, which is due to coils \& magnets. Although structure-wise FSPM resembles switched reluctance machine (SRM), the reluctance torque from coils cannot drive any load including the rotors own mass.

If $i(t)$ is the time dependent current of phase $\mathrm{A}$ and $\lambda(t)$ is the time dependent flux linkage of phase $A$, then for a certain time instant, i.e. at a certain rotor position, the energy function becomes:

$$
W_{c}=f(\lambda(i))
$$

The energy converted into mechanical work is in fact the change in coenergy $W_{c}$. The torque capability of an electrical machine can be calculated by computing the total area enclosed by the $\lambda-i$ loop. Although both $i(t)$ and $\lambda(t)$ are functions of time, the energy conversion loop itself is not dynamic. From this quasi-static loop, the torque capability of the machine can be determined by calculating the average torque $T_{\text {ave }}$ using the following formula [11-12]: $T_{\text {ave }}=\left|\frac{m p}{2 \pi} W_{c}\right|$.

with the phase number $m$ and the pole pair number $p$. For the 12/10 FSPM, $m$ equals 3 and $p$ equals 10 . Each rotor tooth in FSPM acts like a magnet pair in BLDC.

For transient torque calculation as a function of rotation, additional information is required besides the obtained static energy conversion loop. The electromagnetic torque is proportional to the change in the magnetic energy $\Delta W c$; the change from one rotor position to the next one is bound by magnetization curves. The curves show how the inductance changes, if current increases. Each point of the magnetization curve corresponds to the same rotor position of different energy conversion loops, i.e. of different current levels.

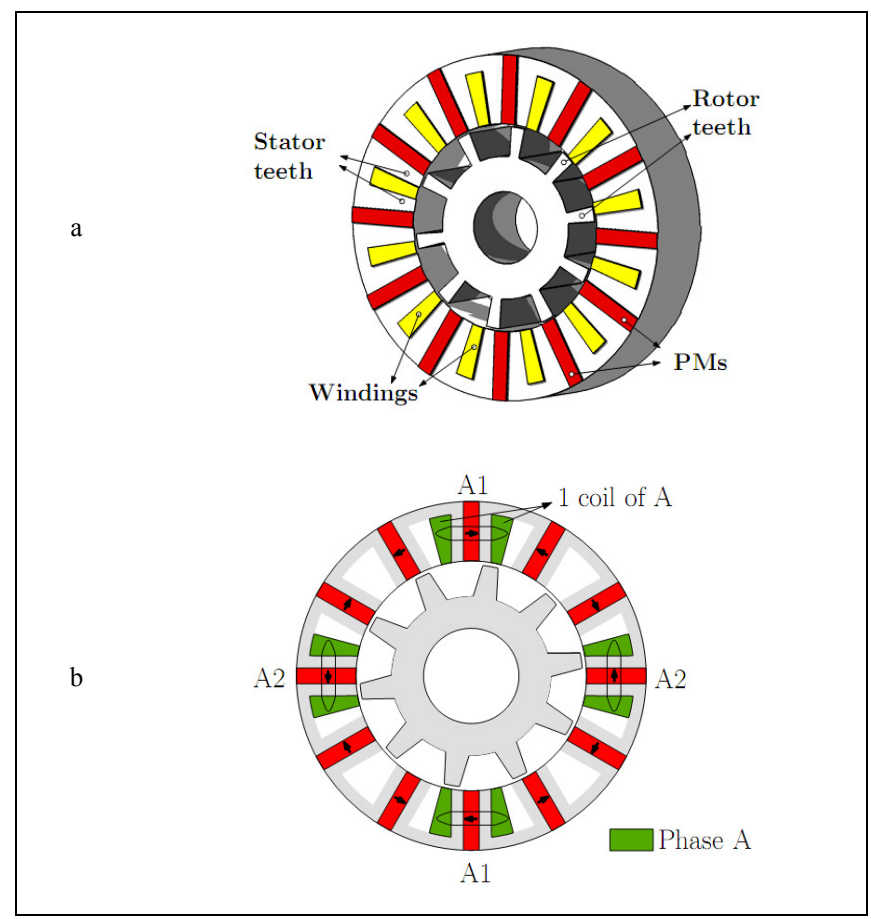

Fig. 1. FSPM with 10 rotor and 12 stator poles.

a) $3 \mathrm{D}$ illustration b) Phase A coil distribution

Figures 4a-b show the change of energy conversion loops with increasing stator current. In Figure 4a, all loops have the same orientation and the loop with a higher current encircles completely the loop with a lower current. Once magnets are included in the calculation, the orientation of the loops in Figure $4 \mathrm{~b}$ change with the increasing current and the increase in the loop area is significantly higher than in Figure 4a. In the first and forth quadrants of Figure $4 \mathrm{~b}$ at the flux linkage upper and lower limit (0-2.5A interval), there exist very small areas where the lower current loop is not covered by the higher current loop. It is a result of the sudden change in the loop orientation with increasing current. Due to magnets the flux linkage changes in this interval at a much faster rate compared to the case with only coils. Visually, a certain rotor position in Figure $4 \mathrm{~b}$ occurs in a shifted position at a higher current level. Therefore, physically the shift does not mean that the higher current loop does not cover an energy region which is covered by the lower current loop.

\section{A. Magnetization curves}

Magnetization curves, which are imperative to transient torque calculation, can be calculated by applying different current values at the same rotor position. Along one magnetization curve, the rotor position stays constant. Examples of magnetization curves are plotted in Figure 4c, when only phase A coils and magnets are activated. The curves 
are bound on the outer side by the energy conversion loop corresponding to the current value supplied to the machine. For the FSPM with coils only, magnetization curves obtained with linear analysis $\left(\mu_{\text {iron }}=\infty\right)$ are identical to the magnetization curves obtained from nonlinear analysis (Figure 4d). On the other hand, these curves differ for the FSPM with coils \& magnets.

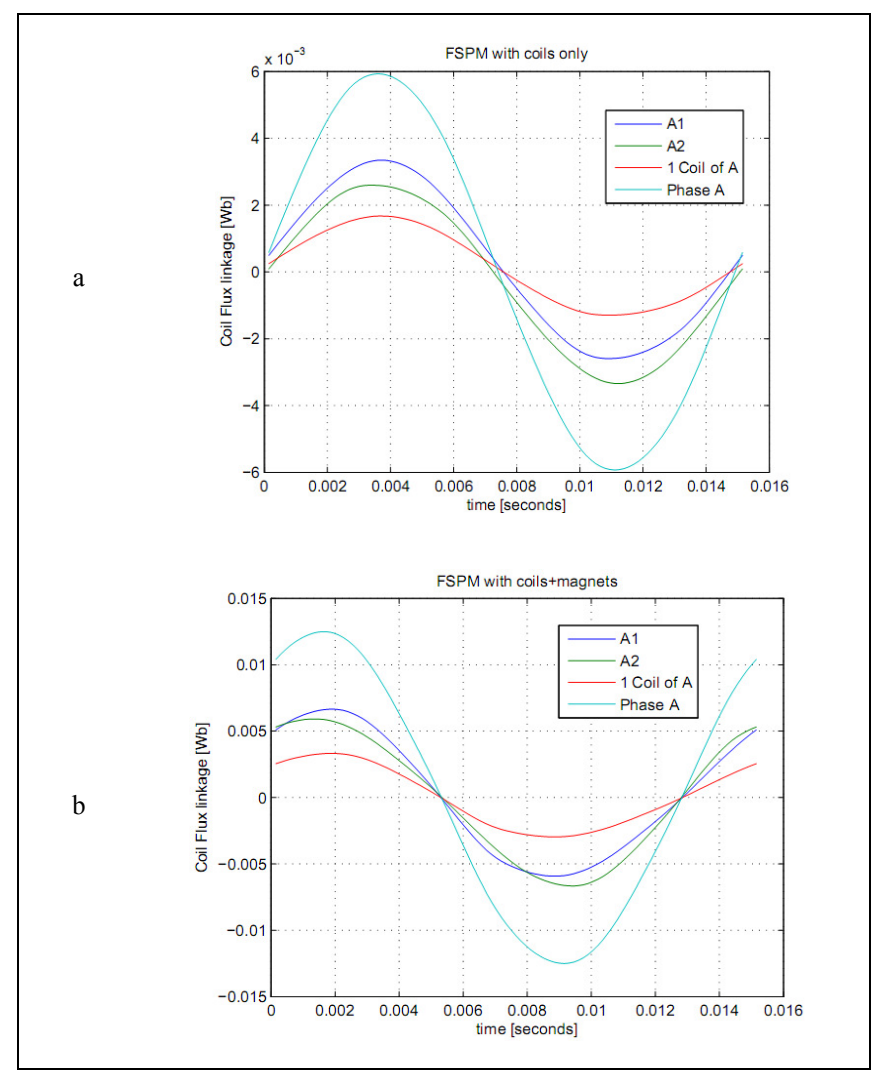

Fig. 2. Flux linkage of different coils of phase A for 11A. a) With coils only b) With coils \& magnets

Once magnets are included in the analysis, the magnetization curves become distinct as illustrated in Figure 4d. For both linear and nonlinear analysis with coils \& magnets, the curves have a shift at initial value $i=0$ corresponding to their PM-flux linkage values $\left(\lambda_{P M}\right)$. Since theoretically the iron is capable to store more magnetic flux in the linear case, $\lambda_{P M}$ value is higher for the linear case compared to the nonlinear. Due to saturation of the iron material, the magnetization curves in Figure $4 \mathrm{~d}$ deviate from each other while the current increases.

Unlike SRM, in FSPM current is not responsible for entering the nonlinear region in the $\lambda-i$ characteristic. With the presence of magnets, FSPM is pre-biased in the magnetization curve up to a point, where it can exchange more magnetic energy. Eventually this high change in the energy storage increases the torque capability of the FSPM significantly compared to a SRM, which has a very similar iron structure compared to FSPM. The use of magnetization curves plays an important role for making such useful comparisons, which provide more insight into the FSPM operation.

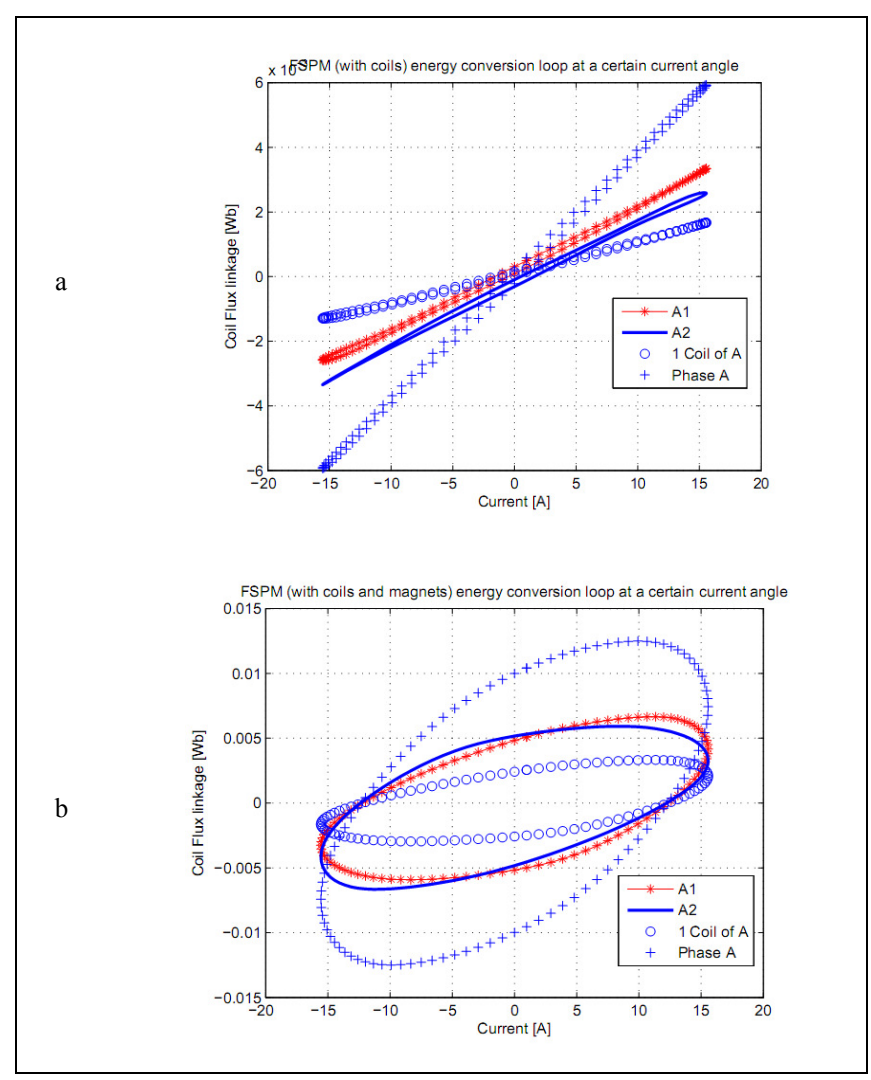

Fig. 3. Energy conversion loops of FSPM for 11A a) With coils only b) With coils \& magnets

\section{TRANSIENT TORQUE CALCULATIONS}

As stated before, torque production depends on the change in the stored magnetic energy $\Delta W_{c}$ with an example given in Figure 5. The energy conversion loop $W_{c}$ in Figure 5a is exaggerated for illustrative purpose. The actual loop in Figure $4 \mathrm{a}$ is much smaller than the illustrated loop in Figure 5a. To calculate the transient torque $T(\theta)$ at constant current, the areas $\Delta W_{c}$ in Figure 5 have to be divided to the mechanical displacements $\Delta \theta_{m}$ :

$$
T(\theta)=\left.\frac{\Delta W_{c}}{\Delta \theta_{m}}\right|_{i=\text { const }}
$$

Using formula (3) gives torque only from one phase. Since FSPM is a three phase machine, the result is shifted electrically $120^{\circ}$ for the remaining two phases. Eventually, resulting torques from all three phases are added together. Preliminary results show that by only using energy conversion loops, obtained transient torques have the right effective value and the correct shape, but not the correct torque ripple amplitude. The torque ripple of the FSPM consists of the cogging torque and 
reluctance torque. It is stated in [9], transient torque can be calculated by using the energy conversion loops. This statement is true but not complete. The transient torque consists of the average torque and the torque ripple. If torque ripple component is only reluctance torque due to the rotor saliency (in case of SRM), the amplitude of transient torque can be calculated by the loop.

However in FSPM, cogging torque is the dominant torque ripple, which is a a result of not only the changing airgap permeance between the rotor and stator iron parts but also the changing magnet related permeances, i.e. inner permeance and leakages. The static energy conversion loop cannot visualize the dynamic change of the magnetic loading, therefore the loop is not able to calculate the ripple correctly. In the paper, energy conversion loops are used to calculate the instantaneous torque, to which cogging torque is added later. How to estimate the cogging torque is discussed in the next subsection more detailed.

Transient torques calculated by the FSPM's energy conversion loops are given in Figures 6-7. The results match to FEM results, where virtual work method is chosen. The magnetization curves for the FSPM with coils only, do not differ for the linear and nonlinear analysis, therefore their transient torques in Figure 6 are identical. The similarity in the results is due to the low energy output, when only coils are considered as the source. The results for the FSPM with coils $\&$ magnets are presented in Figure 7 . In this case, the torque calculations for the linear and nonlinear case show a distinct difference both in magnitude and in ripple shape. The nonlinear iron cannot store as much magnetic energy in the linear case. Therefore, the average torque decreases in the nonlinear case. The area in Figure $5 \mathrm{~b}, \Delta W_{c}$ in the nonlinear analysis, does not change as rapidly as in the linear analysis, which causes a decrease of torque ripple oscillations in the nonlinear case.

The important part is the calculation of the load lines. In practice, these load lines are changing with nonlinear intervals depending on the magnetic circuit and on the magnetic load. Even for steady-state analysis, it is not straight forward to estimate the load lines when the machine is rotating. These can be calculated using reluctance network models presented in [2, 7]. In FSPM, since the whole structure is magnetically prebiased, due to the coupling of energy sources in stator, the magnetic change of load lines could be assumed to be linear. The calculation is done first for one magnet only. Next, the calculations for the similarly oriented magnets is realized, which are labeled as $N$-poles and S-poles as shown in Figure 8.

Finally, the result from two poles is superimposed to calculate one period of cogging torque. Due to the assumption of linear load lines, the estimation of cogging torque is with an error of $25 \%$. In fact, this error exactly corresponds to the ripple calculated by the $\lambda-i$ loop. Summarizing, the $25 \%$ error is the reluctance component of the torque ripple, which depends on the coil inductance. For all the transient torque calculations shown previously, this estimation is added to the dynamic torque calculated by using $\lambda-i$ loop. This analysis also proves that the main torque ripple component of FSPM is due to the magnet-iron interaction, i.e. $75 \%$ of the torque ripple.

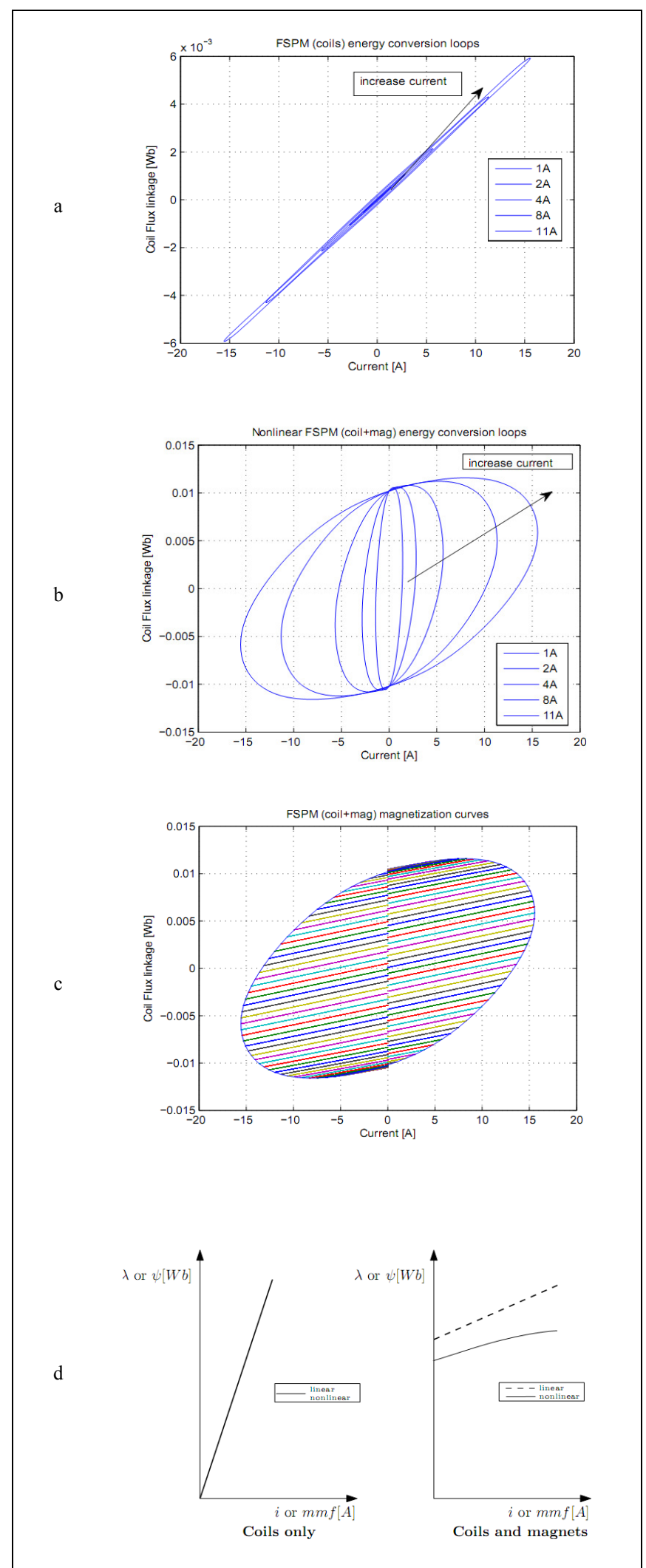

Fig. 4. a-b) Change of energy conversion loops of FSPM with increasing current, c-d) Magnetization curves. a) With coils only b) With coils \& magnets c) Magnetization curves 11A d) Ideal magnetization curves 


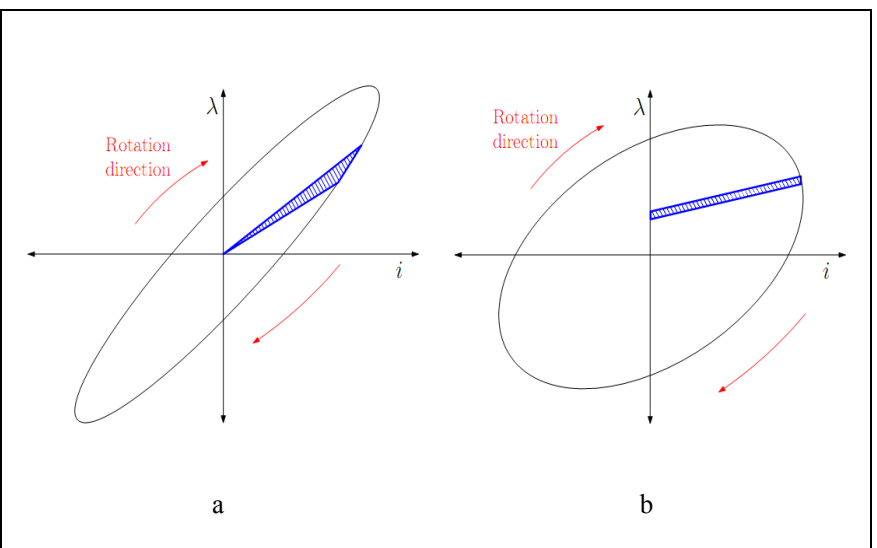

Fig. 5. $\Delta W$ between two consecutive rotor positions.

a) With coils only (enlarged view) b) With coils \& magnets (normal view)

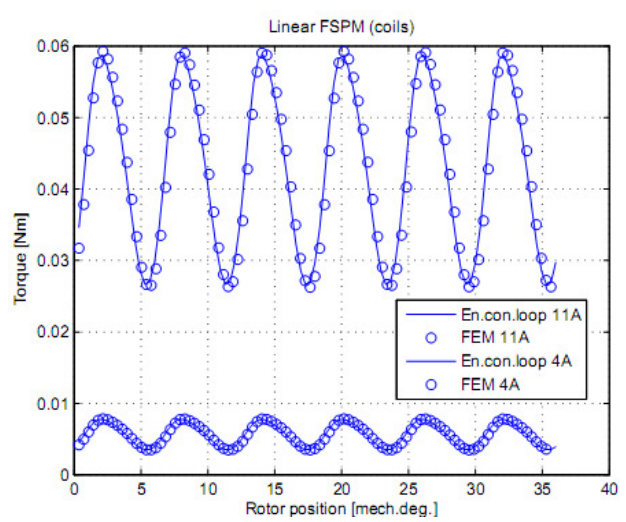

b

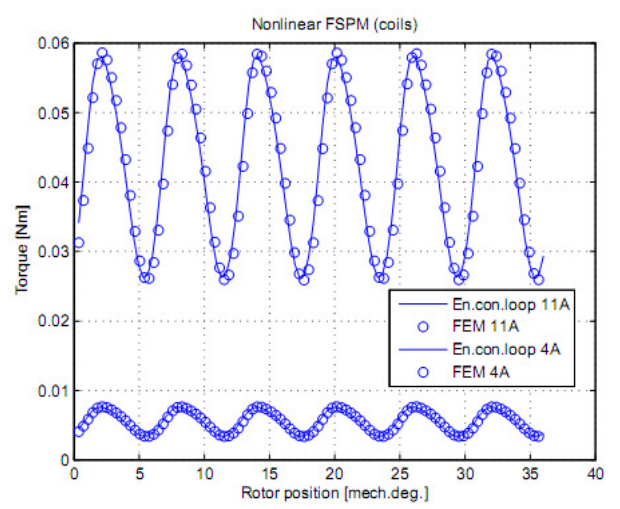

Fig. 6. Torque calculations with coils only. a) Linear analysis b) Nonlinear analysis

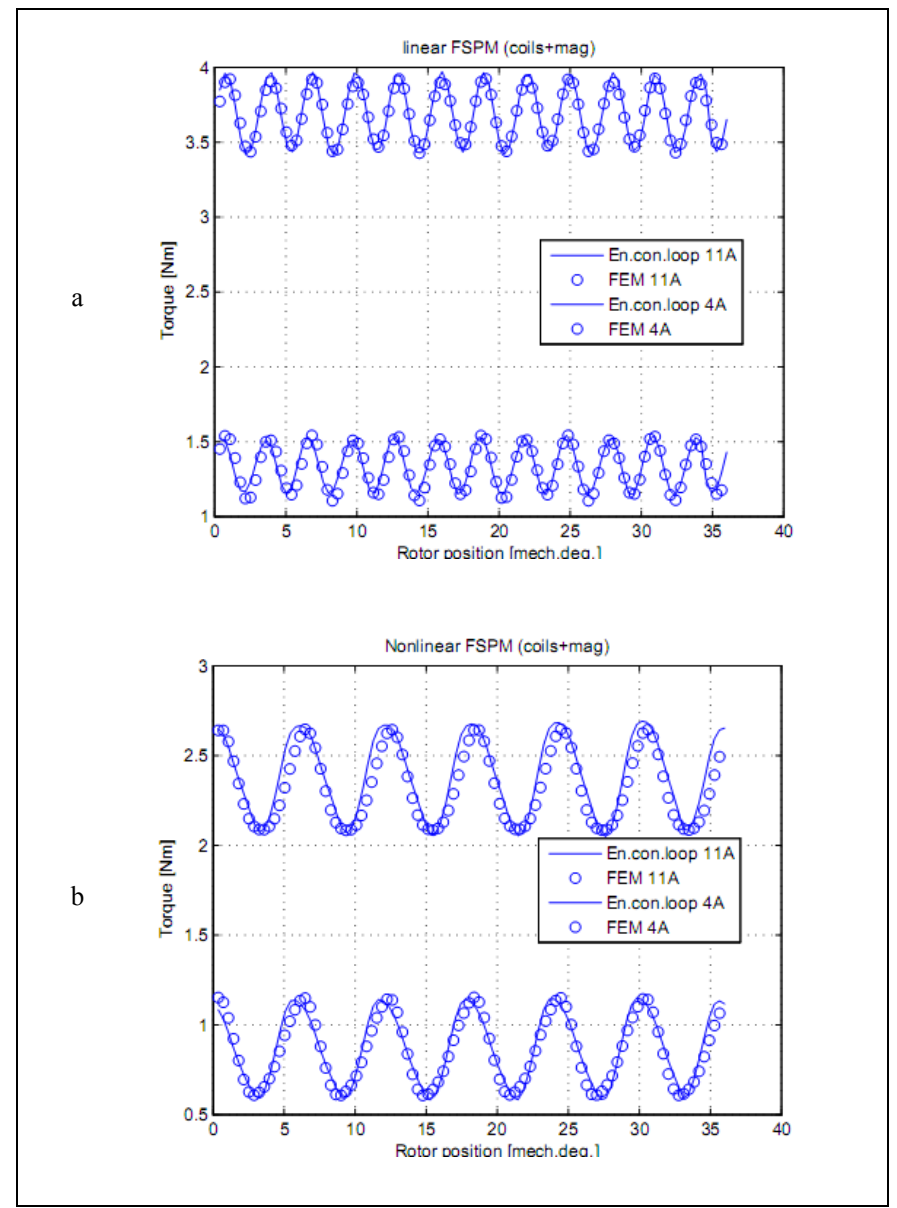

Fig. 7. Torque calculations with coils and magnets. a) Linear analysis b) Nonlinear analysis

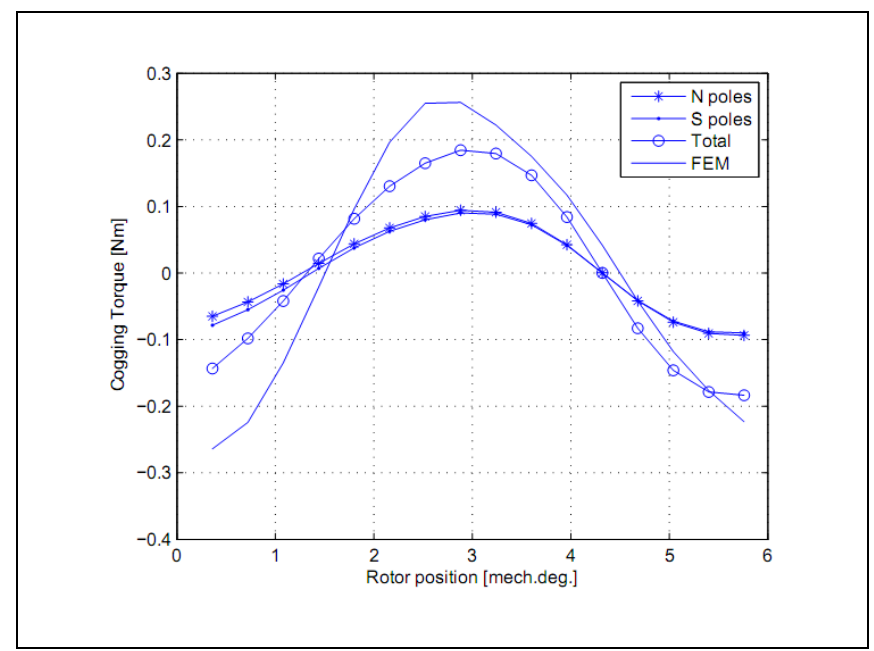

Fig. 8. Cogging torque estimated from $\Phi$-mmf diagram. 


\section{CONCLUSIONS}

All quality electromagnetic designs require an understanding of the system. In automotive, due to the interaction of various subsystems, i.e. electrical, electronic, mechanic, etc., it is sometimes hard to estimate the behavior of each element of these subsystems. Energy management is very dedicated to the limited energy sources on the subsystem level. Precisely at this point, energy conversion loop technique provides the required visual tools to understand the machine operation and estimation of the parameters, from which not only the academic researchers but also the design engineers can benefit.

Since novel flux-switching PM machines require a good understanding of the machine operation, in this work, the energy sources are evaluated separately, as coils \& magnets. Both the linear and nonlinear soft magnetic material properties are evaluated in this paper, of which the nonlinear aspects have often been neglected in the literature. For each defined case, energy conversion loops are created successfully by concluding the physical meaning for the machine operations. Concisely, based on the findings the general concept of energy conversion loops has been extended to cover the FSPM machine class for proper energy management system.

\section{REFERENCES}

[1] T. Heoung Kim, "A Study on the Design of an Inset-Permanent-MagnetType Flux-Reversal Machine", IEEE Transactions on Magnetics, Vol. 45, No. 6, pp.2859-2862, 2009.

[2] E. Ilhan, B. L. J. Gysen, J. J. H. Paulides, E. A. Lomonova, "Analytical Hybrid Model for Flux Switching Permanent Magnet Machines", IEEE Transactions on Magnetics, Vol. 46, No. 6, pp. 1762-1765, 2010.

[3] E. Ilhan, J. Paulides, E. Motoasca, E. Lomonova, "Conformal Mapping: Schwarz-Christoffel Method for Flux-Switching PM
Machines", COMPUMAG 2011, $18^{\text {th }}$ International Conference on the Computation of Electromagnetic Fields, Australia, 2011.

[4] Z. Zhu, J. T. Chen, Y. Pang, D. Howe, S. Iwasaki, R. Deodhar, "Modeling of end-effect in flux-switching permanent magnet machines", ICEMS 2007, International Conference on Electrical Machines and Systems, pp. 943-948, 2007.

[5] B. L. J. Gysen, E. Ilhan, K. J. Meessen, J .J. H. Paulides, E .A. Lomonova, "Modeling of Flux Switching Permanent Magnet Machines With Fourier Analysis", IEEE Transactions on Magnetics, Vol. 46, No. 6, pp. 1499-1502, 2010.

[6] C. Anyuan, R. Nilssen, A. Nysveen, "Analytical design of a high-torque flux-switching permanent magnet machine by a simplified lumped parameter magnetic circuit model", ICEM 2010, XIX International Conference on Electrical Machines, Vol., pp.1-6, France, 2010.

[7] E. Ilhan, J. Paulides, L. Encica, E. Lomonova, "Tooth contour method implementation for the flux-switching PM machines", ICEM 2010, XIX International Conference on Electrical Machines, pp.1-6, France, 2010.

[8] T. J. E. Miller, "Optimal design of switched reluctance motors", IEEE Transactions on Industrial Electronics, Vol. 49, No. 1, pp. 15-27, 2002.

[9] D. Staton, R. Deodhar, W. Soong, T. Miller, "Torque prediction Using the flux-mmf diagram in ac, dc, and reluctance motors", IEEE Transactions on Industry Applications, Vol. 32, No. 1, pp. 180-188, 1996.

[10] M. Cheng, K. Chau, C. Chan, "Static characteristics of a new doubly salient permanent magnet motor", IEEE Transactions on Energy Conversion, Vol. 16, No. 1, pp. 20-25, 2001.

[11] T. Miller, M. McGilp, "Nonlinear theory of the switched reluctance motor for rapid computer-aided design", IEEE Proceedings on Electric Power Applications, Vol. 137, No. 6, pp. 337-347, 1990.

[12] D. Staton, W. Soong, T. Miller, "Unified theory of torque production in switched reluctance and synchronous reluctance motors", IEEE Transactions on Industry Applications, Vol. 31, No. 2, pp. 329-337, 1995.

[13] R. Deodhar, D. Staton, T. Jahns, T. Miller, "Prediction of cogging torque using the flux-mmf diagram technique", IEEE Transactions on Industry Applications, Vol. 32, No. 3, pp. 569-576, 1996. 